http://dx.doi.org/10.35381/s.v.v3i1.453

\title{
Infección del tracto urinario por enterobacterias productoras de betalactamasas de espectro extendido (BLEE)
}

\section{Urinary tract infection by enterobacteria producing extended-spectrum betalactamases (ESBL)}

Fidel Castro González fidel.castro@unesum.edu.ec

Universidad Estatal del Sur de Manabí, Jipijapa

Ecuador

https://orcid.org/0000-0003-1415-5583

Jhon Bryan Mina Ortiz

mina-ortiz-bryan@hotmail.com

Universidad Estatal del Sur de Manabí, Jipijapa

Ecuador

https://orcid.org/0000-0002-3455-2503

Jenniffer Elena Quimis Cañarte

Jennifer.quimis@hotmail.com

Universidad Estatal del Sur de Manabí, Jipijapa

Ecuador

https://orcid.org/0000-0001-9659-7777

Nereida Valero Cedeño

nereida.valero@unesum.edu.ec

Universidad Estatal del Sur de Manabí, Jipijapa

Ecuador

https://orcid.org/0000-0003-3496-8848

Recibido: 6 de septiembre de 2019

Aprobado: 28 de septiembre de 2019

\section{RESUMEN}

Las infecciones del tracto urinario provocadas por enterobacterias productora de betalactamasas de espectro extendido (BLEE) han tomado importancia por altas tasas de morbi-mortalidad a nivel mundial, debido al uso irracional de antibioterapias en pacientes con infecciones recurrentes provocando complicaciones severas como insuficiencia renal crónica. El propósito de esta investigación es puntualizar conocimientos sobre epidemiología, diagnóstico, tratamiento y prevención que puedan ser aplicados en la práctica clínica por corresponder a una temática que abordan todos los países en especial las infecciones provocadas por enterobacterias productoras de BLEE. 
Descriptores: Betalactamasas de espectro extendido; Enterobacterias; Resistencia a antibióticos; Infección; Tracto urinario.

\begin{abstract}
The urinary tract infections caused by producing Enterobacteriaceae lactamase spread spectrum (ESBL) have become important for high rates of morbidity and mortality worldwide, due to irrational use of antibiotic therapies in patients with recurrent infections causing severe complications such as chronic renal failure. The purpose of this research is to point out knowledge of epidemiology, diagnosis, treatment and prevention that can be applied in clinical practice to correspond to a theme that address all countries especially those caused by ESBL-producing Enterobacteriaceae infections.
\end{abstract}

Descriptors: Extended spectrum betalactamases; Enterobacteriaceae; Antibiotic resistance; Infection; Urinary tract.

\title{
INTRODUCCIÓN
}

Las infecciones del tracto urinario (ITU) ocasionadas por enterobacterias productoras de betalactamasas de espectro extendido (BLEE) han adquirido importancia debido a las altas tasas de morbilidad y mortalidad en el mundo por circunstancias referidas al tratamiento empírico que dependerá de la edad y sexo aumentado en tal medida las comorbilidades y clínica del paciente (1).

La resistencia a los antimicrobianos es reconocida por la Organización Mundial de la Salud (OMS) como unas de las principales amenazas para salud pública en todo el mundo con impacto en varias áreas de salud y la sociedad. Por el contrario, muchos países no cuentan con planes para hacer frente a la resistencia a los antimicrobianos al no tener los recursos necesarios que favorezcan la elaboración de soluciones, por ello, la OMS tiene el propósito de elaborar un plan de acción global con la finalidad de reducir las infecciones optimizando el uso de antimicrobianos en base a una lucha sostenible contra la resistencia bacteriana (2).

Las bacterias productoras de BLEE aumentan los costos terapéuticos durante la estancia hospitalaria, debido al uso de antibióticos de elevado valor monetario, porque 
hace años atrás las ITU eran combatidas con eficiencia mediante antimicrobianos comunes de uso hospitalario. En la actualidad la administración descontrolada de fármacos y automedicación ha provocado que estas bacterias generen mecanismo de resistencia haciendo más complicada la antibioterapia, estudios han demostrado mayor susceptibilidad para la adquisición de estos patógenos en aquellos pacientes con trastornos urogenitales relacionados con enfermedades crónicas, anomalías funcionales y factores inmunológicos $(3,4)$.

El uso irracional de antibioterapias en pacientes con infecciones recurrentes del tracto urinario conlleva a complicaciones severas en el riñón como la insuficiencia renal crónica, tal es el caso del gran porcentaje de infecciones urinarias en mujeres por el incorrecto aseo de sus genitales, como consecuencia de colonizaciones bacterianas, donde microrganismos principalmente enterobacterias ascienden por el conducto urinario hasta llegar a la vejiga, lugar diana para ocasionar patogenicidad $(5,6)$.

Las infecciones del tracto urinario han sido clasificadas altas y bajas dependiendo el sitio de focalización, altas cuando está localizada en riñones y uréteres, consideradas más complicadas. Por otra parte, son bajas cuando el sitio blanco es la vejiga y se consideran menos complicadas (7).

Se han evidenciado pocas investigaciones locales que demuestren la importancia de los patógenos productores de BLEE, que se han convertido en causa frecuente de consultas médicas a nivel de atención primaria, cuyo conocimiento, permitirá orientar las acciones de vigilancia epidemiológica y las políticas de salud. El propósito de este artículo de revisión es enfatizar varios aspectos que intervienen en las infecciones urinarias causadas por enterobacterias productoras de BLEE, con la finalidad de puntualizar conocimientos que puedan ser aplicados en las prácticas clínicas para el diagnóstico, tratamiento y prevención de este importante problema de salud pública. Para ello, se realizaron búsquedas bibliográficas focalizadas en infecciones del tracto urinario por enterobacterias productoras de BLEE, en bases de datos de revistas científicas como Pubmed, Scielo, Lilacs, Elsevier y Medigraphic, entre otras. Como palabras clave se utilizaron: enterobacterias productoras de BLEE, infección del tracto urinario por BLEE y se seleccionaron los artículos publicados en un periodo menor a 5 años en relación al año actual. 


\section{DESARROLLO}

\section{Enterobacterias}

La familia Enterobacteriaceae constituye uno de los más grandes grupo de bacilos gramnegativos con importancia clínica, desde el punto de vista clínico producen una gran variedad de enfermedades en el ser humano. Se pueden clasificar en dos grupos de interés hospitalario enterobacterias patógenas primarias (Salmonella entérica, Shigella spp, Yersinia spp, Klebsiella spp y algunas cepas de Escherichia coli (E. coli) y enterobacterias oportunistas (Proteus spp, Serratia spp, Enterobacter spp, Morganella spp y Providencia) (8).

La mayoría pertenece a la vida libre o formando parte de otros organismos, se caracterizan por crecer bajo condiciones aerobias o anaerobias, son oxidasa negativo y fermentadores de azucares (carbohidratos) además son responsables de la mayoría de ITUs, algunas cepas son también causantes de diarrea y en el peor de los casos ocasionan bacteriemias letales (9).

En los últimos años, se han detectado cepas de enterobacterias multirresistentes, relacionadas a diversos factores que han incrementado la diseminación de infecciones hospitalarias durante estancias largas, provocando recidivas que empeoran la salud del paciente (10).

\section{Epidemiología}

La resistencia a los antimicrobianos se ha convertido en un gran problema a nivel mundial aproximadamente 700.000 personas mueren cada año por la severidad de la resistencia con un aumento de consumo global de antibióticos en la medicina humana en un $40 \%$ entre 2000 y 2010 (11).

De acuerdo con modelamientos realizados se estima un incremento continuo de la resistencia hacia el año 2050 que llevaría a 10 millones de personas a morir cada año, se espera que 300 millones de personas mueran prematuramente por causa de este enigma sumado a la gran pérdida entre 60 a 100 billones de dólares en la lucha contra la resistencia a los antimicrobianos (11). 
La prevalencia a nivel mundial de infecciones causadas por bacterias productoras de BLEE con participación de 53 países, 303 hospitales y 86776 pacientes hospitalizados, expresan resultados donde al menos el $34.4 \%$ recibieron un antimicrobiano. Entre los principales prescritos en todo el mundo fueron penicilinas con inhibidores de la betalactamasas, cefalosporinas de tercera generación y fluoroquinolonas (12).

Los carbapenémicos fueron recetados con mayor frecuencia en América Latina y Asia occidental-central, por otra parte, la mayor frecuencia de infecciones asociadas a la atención médica fue más alto en América Latina (11.9\%) (12).

En cuanto la prevalencia de infecciones por microorganismo BLEE corresponde lo siguiente: Este de Europa 37.7\%, Norte de Europa 6.0\%, Sur de Europa 1.5\%, Europa Occidental 7.1\%, África 5.3\%, Este y Sur de Asia 6.5\%, Asia Occidental y Central 13.9\%, Oceanía 6.6\%, América Latina 19.1\%, Norte América 4.3\%, respectivamente (12).

Estudios realizados en Ecuador, refieren que las principales infecciones provocadas por enterobacterias BLEE fueron por Escherichia coli, Klebsiella pneumoniae y Klebsiella oxytoca. En una población de 8718 se encontró una prevalencia del 35\% con incremento gradual del $1.1 \%$ en el 2005 y $5.7 \%$ en el 2009 en pacientes hospitalizados principalmente con ITU (13).

Las distintas organizaciones de Ecuador involucradas en la lucha contra la resistencia a los antimicrobianos se suman al plan global ejecutado por la OMS, bajo la tutela del Grupo Asesor en Vigilancia Integrada de la Resistencia a los Antimicrobianos (AGISAR) donde se buscará dar una respuesta multisectorial en contra de este problema (14).

\section{Betalactamasas de espectro extendido (BLEE).}

Los microorganismos con mayor prevalencia de producción de BLEE son enterobacterias como Klebsiella pneumoniae y Escherichia coli; éstas se caracterizan por presentar resistencia a antibióticos como las cefalosporinas de tercera generación, aztreonam, penicilinas y cefalosporinas de espectro reducido. Además, son incapaces de hidrolizar cefeminas (cefoxitina-cefotetán) y carbapenem, se han definido varias 
enzimas entre las más frecuentes están las TEM denominación por ser aislada por primera vez en Temoniera, SHV se debe a una contracción de sulfhidrilo variable y CTX-M por ser codificadas por plásmidos transferibles (15).

E. coli productora de BLEE está involucrada íntimamente en las infecciones urinarias con concordancias en múltiples estudios realizados en varios países, el genotipo más prevalente en todo el mundo es el CTX-M-1 relacionado a factores de riesgos como hospitalización previa, cateterización y viajes al extranjero $(16,17)$.

K. pneumoniae productora de BLEE es un patógeno por excelencia caracterizado por su alta capacidad de virulencia y resistencia a múltiples fármacos en ITU adquirida en hospitales, pero no de aislamientos en la comunidad, el genotipo principal es el CTXM-15 característico por tener actividad con la cefotaxima, así como también ceftazidima, ceftriazona y cefepime $(18,19)$.

\section{Diagnóstico}

Existen varios métodos para identificar enterobacterias productoras de BLEE entre ellos destacaremos los más habituales: Los métodos fenotípicos se especializan en detectar la producción de enzimas BLEE capaces de hidrolizar las cefalosporinas, inhibidores bectalactámicos y aztreonan. Entre ellos están los métodos habituales en la utilización de inhibidores de betalactamasas, prueba de combinación de discos y técnica de E-test. Por consiguiente los métodos genotípicos se caracterizan por la investigación epidemiológica en brotes, especialmente en la diseminación clonal de diferentes cepas o por la existencia de varios plásmidos entre los constituyentes de una misma cepa. Las técnicas más utilizados son la electroforesis en gel de campos pulsados, perfiles plasmídicos, ribotipificación y otros procedimientos basados en la ampliación de los genes a través de la reacción en cadena de la polimerasa para detectar las diferentes enzimas responsables de la producción de BLEE (SHV, TEM y CTX-M) (20).

El método confirmatorio por difusión de disco establecido por la Clinical and Laboratory Standards Institute (CLSI) conocido como método americano, consiste en comparar los diámetros de los halos de ceftazidima (30ug) con ceftazidima/ácido clavulánico o cefotaxima/ácido clavulánico (30 ug), la diferencia de los halos de los 
discos combinados debe ser mayor a $5 \mathrm{~mm}$ en comparación con los halos de los discos solos (21). Otro método confirmatorio, determinado por la Sociedad francesa de Microbiología, es aquel donde se observa el efecto de expansión que genera el disco inhibidor amoxicilina/ácido clavulánico sobre los halos de ceftazidima, cefotaxima, aztreonan y cefepima cuando están distribuidos a una distancia de $2 \mathrm{~cm}$ del disco inhibidor (22).

En la actualidad, se han desarrollado varios métodos de pruebas comerciales rápidas como in house, basadas en el cambio de pH debido a la hidrólisis del anillo bectalactámico de estos antibióticos, sobre todo cefotaxima el cual es muy sensible a la degradación por las enzimas BLEE (23).

\section{Tratamiento}

La selección de un antimicrobiano de forma empírica puede ser considerada como un reto clínico por factores del antibiótico seleccionado, el cual debe poseer gran afinidad en contra de la bacteria infectante; por lo tanto, la selectividad debe estar basada en la epidemiologia local y regional. Los carbapenémicos como imipenem, meropenem y ertapenem son los fármacos considerados de primera elección en caso de infecciones ocasionadas por enterobacterias BLEE, particularmente en infecciones severas caracterizados por ser estables a la hidrólisis ocasionada por las enzimas BLEE con importante distribución en los tejidos corporales (20).

Un estudio realizado en la población China, demuestra la efectividad de carbapenémicos especialmente imipenem, ertapenem y amikacina como agentes efectivos contra las infecciones urinarias causadas por E. coli y K. pneumoniae (24). Otra alternativa terapéutica adecuada es el uso de piperacilina/tazobactam en infecciones por E. coli productora de BLEE tipo CTX-M, dicha investigación presentó gran sensibilidad de esta enterobacteria, en particular a este antimicrobiano, especialmente de origen de ITUs (25).

En otro estudio, demostraron la posibilidad de un sinergismo como consecuencia de la combinación de meropenem con fosfomicina, el cual logra la supresión de los clones resistentes a fosfomicina; resultado que podría ser prometedor en infecciones complicadas por E. Coli multidrogoresistente (MDR) (26). 
La combinación de colistín con carbapenémicos presenta efecto sinérgico en cepas de $K$. pneumoniae resistentes a ambos antibióticos de combinación, como consecuencia de esta prueba in vitro se expresa actividad bactericida en contra de aquellas cepas productoras de BLEE (27).

\section{Prevención}

Las medidas de control de transmisión de microorganismos hospitalarios pueden clasificarse en cuatro áreas: precauciones estándar, precauciones específicas, medidas de limpieza-desinfección ambiental y actividad de vigilancia. El correcto uso de guantes y la higiene de manos son las principales medidas para prevenir las diseminaciones de microorganismos multirresistentes por lo que se requieren de actividades de formación continuas a través de la vigilancia epidemiológica regional y local. Otras estrategias son el tratamiento de descolonización previa a cirugías en pacientes con catéter venoso central o sometidos a ventilación mecánica y la higiene corporal con clorhexidina (28).

Medidas específicas son la creación de estrategias de comunicación mediante una serie de reuniones con participación de la comunidad y profesionales sanitarios de los hospitales donde se compartan conocimientos sobre los mecanismos de transmisión y estrategias de precaución en caso de brotes epidemiológicos (29).

Por otro lado, la vigilancia epidemiológica hospitalaria es uno de los pilares fundamentales en la disminución de microorganismos MDR, con la identificación temprana de mecanismos de resistencia en pacientes colonizados o infectados por enterobacterias portadores de enzimas con resistencia a los antimicrobianos a través del hisopado rectal (30).

\section{CONCLUSIONES}

La resistencia a los antimicrobianos es una temática que abordan todos los países del mundo, en especial las infecciones provocadas por enterobacterias productoras de BLEE. Al ser parte habitual de la microbiota intestinal en seres humanos, aumentan las posibilidades de contraer infecciones principalmente del tracto urinario, patología frecuente en los hospitales, lo que limita el uso de cefalosporinas y deja pocas 
alternativas terapéuticas disponibles. Esto consecuentemente eleva los costos hospitalarios y las tasas de morbilidad y mortalidad. Por ello, es necesario implementar programas basados en intervenciones en medidas de control que puedan reducir la diseminación de microorganismos resistentes a múltiples fármacos. Una de las soluciones para reducir el número de ITUs por enterobacterias BLEE es implementar programas que regulen el uso indiscriminado de antibióticos como cefalosporinas de tercera generación, carbapenémicos u otros antibióticos capaces de inducir resistencia. Es necesario tomar conciencia sobre la resistencia antimicrobiana cuyo problema puede agravarse en el futuro por la carencia de antibióticos para combatirlas, países como Ecuador deben fortalecer programas educativos sobre medidas de prevención tanto para infecciones nosocomiales como las comunitarias capaces de aumentar la morbi-mortalidad en países en subdesarrollos por falta de inversión económica por el ente regulador. El conocimiento de la epidemiologia local y regional por parte de los profesionales en salud es fundamental porque se podrán crear recomendaciones y criterios en base a las características clínicas del paciente, comportamiento del microorganismo patógeno y actividades de aseguramiento para el uso correcto de antimicrobianos.

\section{REFERENCIAS CONSULTADAS}

1. Alviz Amador A, Gamero Tafur K, Gamero Tafur J, Caraballo Marimon R. Prevalencia de infección del tracto urinario, uropatógenos y perfil de susceptibilidad en un hospital de Cartagena, Colombia. 2016. Rev. Fac. Med. 2018; 63(3): p. 313-317.

2. World Health Organization. Worldwide country situation analysis: response to antimicrobial resistance. [Online].; 2015 [cited 2019 Julio 17. Available from:

https://apps.who.int/iris/bitstream/handle/10665/163468/9789241564946_e ng.pdf\&ua =1;jsessionid=AE34A3CD0916B6D403D6A643D2AB124D?sequ ence $=1$.

3. Będzichowska A, Przekora J, Stapińska Syniec A, Guzek A, Murawski P, Jobs $\mathrm{K}$, et al. Frequency of infections caused by ESBL-producing bacteria in a pediatric ward - single-center five-year observation. Arch Med Sci. 2019; 15(3): p. 688-693. 
4. Pachay Solórzano. Las infecciones bacterianas y su resistencia a los antibióticos. Caso de estudio: Hospital Oncológico "Dr. Julio Villacreses Colmont Solca", Portoviejo. Revista Científica de la Universidad de Cienfuegos. 2018; 10(5): p. 219-223.

5. Majeed H, Aljabani A. Antibiotic Susceptibility Patterns and Prevalence of Some Extended Spectrum Beta-Lactamases Genes in Gram-Negative Bacteria Isolated from Patients Infected with Urinary Tract Infections in AlNajaf City, Iraq. Avicenna Journal of Medical Biotechnology. 2019; 11(2): p. 192-201.

6. Chafla Martínez P, Cerón Pantoja E, Ortíz Zayas E. Infecciones del tracto urinario. La Ciencia al Servicio de la Salud y la Nutrición. 2018; 9(1): p. 2027.

7. Mueses Guerrero Y, Paz Montañez J, Restrepo Restrepo J, Ortiz Martínez $\mathrm{R}$, Acosta Aragón M. Factores asociados y descripción general de infección del tracto urinario en niños. Revista Colombiana Salud Libre. 2016; 11(2): p. 165-171.

8. Pérez Guerrero, Galán Sánchez, Gutiérrez Saborido, Guerrero Lozano. Infecciones por enterobacterias. Medicine - Programa de Formación Médica Continuada Acreditado. 2014; 11(55): p. 3276-328.

9. Kang E, Crouse A, Chevallier L, Pontier S, Alzahrani A, Silué N, et al. Enterobacteria and host resistance to infection. Mammalian Genome. 2018; 29(7): p. 558-576.

10. Remache Cevallos W, Flores Sangacha J. Prevalencia de enterobacterias productoras de carbapenemasas en pacientes de UCI DEL HGDC 20172018. Tesis para Bioquímica Clínica. Quito: Universidad Central del Ecuador, Facultad de Ciencias Químicas; 2019.

11. O'Neill J. Antimicrobial Resistance: Tackling a crisis for the health and wealth of nations. Antimicrobial Resistance. 2014.

12. Versporten A, Zarb P, Caniaux I, Françoise Gros M, Drapier N, Miller M, et al. Antimicrobial consumption and resistance in adult hospital inpatients in 53 countries: results of an internet-based global point prevalence survey. The Lancet Global Health. 2018; 6(6): p. 619-629.

13. León Astudillo C, Pacheco Quito M. Epidemiología de las infecciones por microorganismos productores de BLEE en el Hospital Vozandes Quito entre los años 2005 y 2009. Tesis Doctoral. Cuenca: Universidad del Azuay, Facultad de Medicina; 2010. 
14. OMS. OPS/OMS apoya a Ecuador en la respuesta multisectorial a la resistencia a los antimicrobianos. [Online].; 2018 [cited 2019 Julio 19. Available from: https://www.paho.org/ecu/index.php?option=com content\&view=article\&id $=2070$ :ops-oms-apoya-a-ecuador-en-la-respuesta-multisectorial-a-laresistencia-a-los-antimicrobianos\&ltemid=360.

15. Livermore. Beta-lactamases in laboratory and clinical resistance. Clin Microbiol Rev. 1995; 8(4): p. 557-584.

16. Al-Jamei S, Albsoul A, Bakri F, Al-Bakri A. Extended-spectrum-lactamase producing $\mathrm{E}$. coli in urinary tractinfections: A two-center, cross-sectional study of prevalence,genotypes and risk factors in Amman, Jordan. Journal of Infection and Public Health. 2019; 12(1): p. 21-25.

17. Brolund, Edquist, Makitalo, Olsson Liljequis, Soderblom, Tegmark Wisell, et al. Epidemiology of extended-spectrum b-lactamase-producing Escherichia coli in Sweden 2007-2011. Clin Microbiol Infect. 2014; 20(6): p. 344-352.

18. Caneiras C, Lito L, Melo Cristino J, Duarte A. Community- and HospitalAcquired Klebsiella pneumoniae Urinary Tract Infections in Portugal: Virulence and Antibiotic Resistance. Microorganisms. 2019; 16(5): p. 1-14.

19. Olusoga Ogbolu D, Terry Alli O, Webber M, Sunday Oluremi A, Moses Oloyede O. CTX-M-15 is Established in Most Multidrug-Resistant Uropathogenic Enterobacteriaceae and Pseudomonaceae from Hospitals in Nigeria. Eur J Microbiol Immunol (Bp). 2018; 8(1): p. 20-24.

20. Aguilar Zapata D. E. coli BLEE, la enterobacteria que ha atravesado barreras. Rev Invest Med Sur Mex. 2015; 22(2): p. 57-63.

21. Institute CaLS. Performance Standards for Antimicrobial Susceptibility Testing; Twenty-First Informational Supplement. CLSI document M100-S21. $2011 ; 31(1)$.

22. Perú MdSd. Manual de procedimientos para la prueba de sensibilidad antimicrobiana por el método de disco difusión. Serie de normas técnicas $N^{\circ}$ 30. Lima: Instituto Nacional de Salud, Organismo Público Descentralizado de Sector Salud; 2002.

23. Jiménez Guerra G, Hoyos Mallecot $Y$, Rodríguez Granger J, Navarro Marí J, Gutiérrez Fernández J. Método rápido para la detección de la sensibilidad a cefotaxima en enterobacterias. Rev Argent Microbiol. 2016; 48(3): p. 320324. 
24. Zhang H, Johnson A, Zhang G, Yang Y, Zhang J, Li D, et al. Susceptibilities of Gram-negative bacilli from hospital- and community-acquired intraabdominal and urinary tract infections: a 2016-2017 update of the Chinese SMART study. Infection and Drug Resistance. 2019; 24(12): p. 905-914.

25. Álvarez J, Rojas Á, Carvajal C, Revello J, Meza P, Guggiana P, et al. Evaluación de susceptibilidad y respuesta al tratamiento con piperacilina/tazobactam en pacientes con infecciones por Escherichia coli productoras de $\beta$-lactamasas de espectro extendido (BLEE) CTX-M. Revista chilena de infectología. 2018; 35(4): p. 343-350.

26. Docobo Perez F, Drusano G, Johnson A, Goodwin J, Whalley S, Ramos Martin V, et al. Pharmacodynamics of Fosfomycin: Insights into Clinical Use for Antimicrobial Resistance. Antimicrob Agents Chemother. 2015; 59(9): p. 5602-610.

27. Herrera S, Guillermo R. Actividad in vitro de la combinación de colistín con diferentes antibióticos contra cepas de Acinetobacter baumannii resistentes a carbapenémicos y klebsiella pneumoniae productoras de $\beta$-lactamasas de espectro extendido en algunos hospitales de Chile. Salud. 2016.

28. Lupión C, López Cortés L, Rodríguez Baño J. Medidas de prevención de la transmisión de microorganismos entre pacientes hospitalizados. Higiene de manos. Enfermedades Infecciosas y Microbiología Clínica. 2014; 32(9): p. 603-609.

29. Fernández Prada M, Martínez Ortega C, Santos Simarro G, Morán Álvarez P, Fernández Verdugo A, Costa Romero M. Brote de Klebsiella pneumoniae productora de betalactamasas de espectro extendido en una unidad de cuidados intensivos neonatales: factores de riesgo y medidas de prevención clave para su erradicación en tiempo récord. Anales de Pediatría. 2019; 91(1): p. 13-20.

30. González Estrada A, Fernández Prada M, Martínez Ortega C, Lana Pérez A, López González M. ICumplimiento de las precauciones de aislamiento de contacto por microorganismos multirresistentes en un hospital de tercer nivel. Revista de Calidad Asistencial. 2015 Octubre; 31(5): p. 51.

\section{REFERENCES CONSULTED}


1. Alviz Amador A, Gamero Tafur K, Gamero Tafur J, Caraballo Marimon R. Prevalence of urinary tract infection, uropathogens and susceptibility profile in a hospital in Cartagena, Colombia. 2016. Rev. Fac. Med. 2018; 63 (3): p. 313317.

2. World Health Organization. Worldwide country situation analysis: response to antimicrobial resistance. [On-line].; 2015 [cited 2019 July 17. Available from: https://apps.who.int/iris/bitstream/handle/10665/163468/9789241564946 eng. pdf\&ua=1;jsessionid=AE34A3CD0916B6D403D6A643D2AB124D? sequence $=$ 1 .

3. Będzichowska A, Przekora J, Stapińska Syniec A, Guzek A, Murawski P, Jobs $\mathrm{K}$, et al. Frequency of infections caused by ESBL-producing bacteria in a pediatric ward - single-center five-year observation. Arch Med Sci. 2019; 15 (3): p. 688-693.

4. Pachay Solórzano. Bacterial infections and their resistance to antibiotics. Case study: "Dr. Oncology Hospital Julio Villacreses Colmont Solca", Portoviejo. Scientific Magazine of the University of Cienfuegos. 2018; 10 (5): p. 219-223.

5. Majeed H, Aljabani A. Antibiotic Susceptibility Patterns and Prevalence of Some Extended Spectrum Beta-Lactamases Genes in Gram-Negative Bacteria Isolated from Patients Infected with Urinary Tract Infections in Al-Najaf City, Iraq. Avicenna Journal of Medical Biotechnology. 2019; 11 (2): p. 192-201.

6. Chafla Martínez P, Cerón Pantoja E, Ortíz Zayas E. Urinary tract infections. Science at the Service of Health and Nutrition. 2018; 9 (1): p. 20-27.

7. Mueses Guerrero Y, Paz Montañez J, Restrepo Restrepo J, Ortiz Martínez R, Acosta Aragón M. Associated factors and general description of urinary tract infection in children. Colombian magazine Salud Libre. 2016; 11 (2): p. 165-171.

8. Pérez Guerrero, Galán Sánchez, Gutiérrez Saborido, Guerrero Lozano. Enterobacterial infections. Medicine - Accredited Continuing Medical Training Program. 2014; 11 (55): p. 3276-328.

9. Kang E, Crouse A, Chevallier L, Pontier S, Alzahrani A, Silue N, et al. Enterobacteria and host resistance to infection. Mammalian Genome 2018; 29 (7): p. 558-576.

10. Rivet Cevallos W, Flores Sangacha J. Prevalence of carbapenemases producing enterobacteria in ICU patients of the HGDC 2017-2018. Thesis for Clinical Biochemistry. Quito: Central University of Ecuador, Faculty of Chemical Sciences; 2019. 
11. O'Neill J. Antimicrobial Resistance: Tackling a crisis for the health and wealth of nations. Antimicrobial Resistance 2014.

12. Versporten A, Zarb P, Caniaux I, Françoise Gros M, Drapier N, Miller M, et al. Antimicrobial consumption and resistance in adult hospital inpatients in 53 countries: results of an internet-based global point prevalence survey. The Lancet Global Health. 2018; 6 (6): p. 619-629.

13. León Astudillo C, Pacheco Quito M. Epidemiology of infections by BLEE producing microorganisms at Vozandes Quito Hospital between 2005 and 2009. Doctoral thesis. Cuenca: University of Azuay, Faculty of Medicine; 2010.

14. WHO. PAHO / WHO supports Ecuador in the multisectoral response to antimicrobial resistance. [On-line].; 2018 [cited 2019 July 19. Available from: https://www.paho.org/ecu/index.php?option=com_content\&view=article\&id=20 70:ops-oms-apoya-a-ecuador-en-la-respuesta- multi-sector-to-resistance-toantimicrobials \& Itemid $=360$.

15. Livermore. Beta-lactamases in laboratory and clinical resistance. Clin Microbiol Rev. 1995; 8 (4): p. 557-584.

16. Al-Jamei S, Albsoul A, Bakri F, Al-Bakri A. Extended-spectrum-lactamase producing $\mathrm{E}$. coli in urinary tractinfections: A two-center, cross-sectional study of prevalence,genotypes and risk factors in Amman, Jordan. Journal of Infection and Public Health. 2019; 12(1): p. 21-25.

17. Brolund, Edquist, Makitalo, Olsson Liljequis, Soderblom, Tegmark Wisell, et al. Epidemiology of extended-spectrum b-lactamase-producing Escherichia coli in Sweden 2007-2011. Clin Microbiol Infect. 2014; 20(6): p. 344-352.

18. Caneiras C, Lito L, Melo Cristino J, Duarte A. Community- and HospitalAcquired Klebsiella pneumoniae Urinary Tract Infections in Portugal: Virulence and Antibiotic Resistance. Microorganisms. 2019; 16(5): p. 1-14.

19. Olusoga Ogbolu D, Terry Alli O, Webber M, Sunday Oluremi A, Moses Oloyede O. CTX-M-15 is Established in Most Multidrug-Resistant Uropathogenic Enterobacteriaceae and Pseudomonaceae from Hospitals in Nigeria. Eur $\mathrm{J}$ Microbiol Immunol (Bp). 2018; 8(1): p. 20-24.

20. Aguilar Zapata D. E. coli BLEE, la enterobacteria que ha atravesado barreras. Rev Invest Med Sur Mex. 2015; 22(2): p. 57-63.

21. Institute CaLS. Performance Standards for Antimicrobial Susceptibility Testing; Twenty-First Informational Supplement. CLSI document M100-S21. 2011; 31(1). 
22. Perú MdSd. Manual de procedimientos para la prueba de sensibilidad antimicrobiana por el método de disco difusión. Serie de normas técnicas $\mathrm{N}^{\circ}$ 30. Lima: Instituto Nacional de Salud, Organismo Público Descentralizado de Sector Salud; 2002.

23. Jiménez Guerra G, Hoyos Mallecot Y, Rodríguez Granger J, Navarro Marí J, Gutiérrez Fernández J. Método rápido para la detección de la sensibilidad a cefotaxima en enterobacterias. Rev Argent Microbiol. 2016; 48(3): p. 320-324.

24. Zhang H, Johnson A, Zhang G, Yang Y, Zhang J, Li D, et al. Susceptibilities of Gram-negative bacilli from hospital- and community-acquired intra-abdominal and urinary tract infections: a 2016-2017 update of the Chinese SMART study. Infection and Drug Resistance. 2019; 24(12): p. 905-914.

25. Álvarez J, Rojas Á, Carvajal C, Revello J, Meza P, Guggiana P, et al. Evaluación de susceptibilidad y respuesta al tratamiento con piperacilina/tazobactam en pacientes con infecciones por Escherichia coli productoras de $\beta$-lactamasas de espectro extendido (BLEE) CTX-M. Revista chilena de infectología. 2018; 35(4): p. 343-350.

26. Docobo Perez F, Drusano G, Johnson A, Goodwin J, Whalley S, Ramos Martin $V$, et al. Pharmacodynamics of Fosfomycin: Insights into Clinical Use for Antimicrobial Resistance. Antimicrob Agents Chemother. 2015; 59(9): p. 5602610.

27. Herrera S, Guillermo R. Actividad in vitro de la combinación de colistín con diferentes antibióticos contra cepas de Acinetobacter baumannii resistentes a carbapenémicos y klebsiella pneumoniae productoras de $\beta$-lactamasas de espectro extendido en algunos hospitales de Chile. Salud. 2016.

28. Lupión C, López Cortés L, Rodríguez Baño J. Medidas de prevención de la transmisión de microorganismos entre pacientes hospitalizados. Higiene de manos. Enfermedades Infecciosas y Microbiología Clínica. 2014; 32(9): p. 603609.

29. Fernández Prada M, Martínez Ortega C, Santos Simarro G, Morán Álvarez P, Fernández Verdugo A, Costa Romero M. Brote de Klebsiella pneumoniae productora de betalactamasas de espectro extendido en una unidad de cuidados intensivos neonatales: factores de riesgo y medidas de prevención clave para su erradicación en tiempo récord. Anales de Pediatría. 2019; 91(1): p. 13-20.

30. González Estrada A, Fernández Prada M, Martínez Ortega C, Lana Pérez A, López González M. ICumplimiento de las precauciones de aislamiento de contacto por microorganismos multirresistentes en un hospital de tercer nivel. Revista de Calidad Asistencial. 2015 Octubre; 31(5): p. 51. 
(C2019 por los autores. Este artículo es de acceso abierto y distribuido según los términos y condiciones de la licencia Creative Commons Atribución-NoComercial-Compartirlgual 4.0 Internacional (CC BY-NC-SA 4.0) (https://creativecommons.org/licenses/by-nc-sa/4.0/). 